\title{
FORMACIÓN DE VALORES MORALES EN \\ LA ENSEÑANZA APRENDIZAJE DEL ÁREA \\ "PERSONA, CULTURA Y NATURALEZA"
}

\author{
Isabel Martínez Melgara[5], \\ Martha Zamora Mora ${ }^{[6]}$ \\ Sandra Davis $\mathbf{R}^{[7]}$
}

\section{Resumen}

La investigación: Formación de valores morales en la enseñanza del área: "Persona, Cultura y Naturaleza", 5to. grado, colegio "Marvin Mitchel”, municipio Bilwi, tiene el propósito contribuir en la calidad pertinente de dicho proceso educativo.

Se aborda esta temática para analizar el comportamiento en cuanto a los temas y cómo se practican en los procesos de interacción social entre los propios estudiantes y en relación con los docentes, ya que se percibía que los educandos presentaban problemas de disciplina.

Fue un estudio cualitativo, de corte transversal. Para la obtención de la información requerida, se aplicaron las técnicas siguientes: entrevistas, diálogos y observación a la labor docente de esta asignatura. Además, se realizaron observaciones a estudiantes durante los diferentes espacios en el cual interactúan: receso, aula de clase.

Este estudio servirá para promover el desarrollo de actitudes de solidaridad, cooperación, compañerismo, y como un aporte valioso de consulta a los docentes, a la dirección del centro escolar "Marvin Michell”, MINED, Secretaría de Educación del Gobierno Regional y otras instituciones, al poder disponer de información valiosa para revisar y readecuar los textos, programas educativos relacionados con el área de Persona, Cultura y Naturaleza en Educación Primaria.

Palabras claves: Educación Intercultural Bilingüe (EIB), Ministerio de Educación (MINED), Sistema Educativo Autonómico Regional (SEAR), Valores morales, cultura y naturaleza.

[5] Licda. Educación Intercultural Bilingüe, URACCAN, Bilwi

[6] Licda. Educación Intercultural Bilingüe, URACCAN, Bilwi

[7] Master en Antropología Social, Directora del Instituto de Estudios y Promoción de la Autonomía, URACAAN, diriangenus@yahoo.com 


\section{CIENCIAS SOCIALES}

\section{Introducción}

Desde hace aproximadamente cinco años, se imparte en la Educación Primaria particularmente en Centros de Educación Intercultural Bilingüe, el área de Persona, Cultura y Naturaleza que integra aspectos sobre Estudios Sociales, Ciencias Naturales y Formación Cívica.

En este estudio se analiza la formación de valores morales en la enseñanza del área: Persona, Cultura y Naturaleza. Se seleccionó la escuela "Marvin Mitchell" con estudiantes del 5 to. grado de primaria, por ser uno de los centros de Educación Bilingüe en la localidad que alberga estudiantes multilingües y multiculturales en la ciudad de Bilwi, durante el II semestre del año lectivo 2009.

Esta área promueve el rescate cultural tradicional de las comunidades indígenas y étnicas de la Costa Caribe nicaragüense. Su finalidad fundamental es valorar y respetar los derechos lingüísticos, culturales; conocer y valorar la diversidad cultural; investigar y sistematizar los conocimientos de la tradición del desarrollo musical. Desde estos propósitos el Ministerio de Educación pretende inculcar actitudes morales, parte importante de la vida espiritual e ideológica de la sociedad.

Es importante destacar que el área de estudio en mención es portadora de valores humanos positivos que desarrolla la personalidad, el pensamiento, conducta integral de la niñez en edad escolar.

Debido a lo poco que se hace desde las aulas de clase, en términos de establecer enfoques como la interculturalidad, equidad de género, educación para la paz, cuidado del medio ambiente, conservación del acervo cultural, establecidos en el Sistema Educativo Autonómico Regional, a través de este estudio se ha propuesto saber en qué medida los contenidos que se brindan en el área de Persona, Cultura y Naturaleza están impactando en la actitud moral de los estudiantes de 5to grado de la escuela "Marvin Mitchell".

Desde la fundación del centro escolar en el año 2001, en la ciudad de Bilwi, se brinda este servicio educativo de manera gratuita a todos los estudiantes; por otro lado, se conoce que este centro ha presentado dificultades en la entrega de textos educativos de parte del MINED, en sus siete años de funcionamiento. Entre ellos, por supuesto, los textos del área de Persona, Cultura y Naturaleza. Entre las consecuencias a estas dificultades existen estudiantes que presentan actitudes relacionadas con la indisciplina, falta de práctica de valores morales positivos tanto en el aula como en otros espacios de la escuela.

El área de Persona, Cultura y Naturaleza se comenzó a implementar en las escuelas bilingües en la Costa Caribe nicaragüenses y en las Escuelas Normales después de la 
transformación curricular en 1998. Esta es un área Integrada por las siguientes subáreas: Recursos Naturales, Historia y Sociedad, Ciencias y Tecnología. Se elaboraron programas y textos denominados: Persona, Cultura y Naturaleza para estudiantes de 4to. Y 5 to. grado en la escuela "Marvin Michell". El programa de esta asignatura consta de cuatro (4) unidades distribuidas de la siguiente manera, Área: Persona, Cultura y Naturaleza[8]:

Antes esta área se impartía de manera aislada, es decir, existían textos separados para cada sub-área: Ciencias Naturales, Formación Cívica y Urbanidad, Geografía e Historia.

Como docentes del área de Persona, Cultura y Naturaleza se ha identificado la importancia de promover contenidos culturales relevantes para la vida y la convivencia, de manera especial el modelo de ciudadanos que demanda la sociedad. En este sentido, se ha definido como objetivo contribuir al fortalecimiento del proceso de enseñanza de los contenidos del área de Persona, Cultura y Naturaleza para la formación de actitudes morales.

\section{Revisión de literatura}

\section{Proceso enseñanza-aprendizaje}

Según Phillipe Perrenoud (2007:53), en el proceso educativo hay que valorar las posibilidades de realización del estudiante en un momento determinado, dependiendo del estadio de desarrollo operatorio en que se encuentre (según las teorías de J. Piaget). La concreción curricular que se haga ha de tener en cuenta estas posibilidades, no tan sólo en referencia a la selección de los objetivos y de los contenidos, sino también en la manera de planificar las actividades de aprendizaje, de forma que se ajusten a las peculiaridades de funcionamiento de la organización mental del estudiante.

Otro aspecto que considera el mismo autor es el conjunto de conocimientos previos que han construido en sus experiencias, escolares y de otras experiencias. El estudiante que inicia un nuevo aprendizaje escolar lo hace a partir de los conceptos, representaciones y conocimientos que ha construido en su experiencia previa y los utilizará como instrumentos de lectura e interpretación que condicionan el resultado del aprendizaje. Este principio especial ha de establecerse en las secuencias del aprendizaje, también tiene implicaciones para la metodología de enseñanza y para la evaluación.

Además, propone que se ha de establecerse una diferencia entre lo que el estudiante es capaz de hacer y aprender sólo y lo que es capaz de hacer y aprender con

[8] Ministerio de Educación, Cultura y Deporte (Mecd; 2003). Programa del área Persona, Cultura y Naturaleza 5to. grado. 
ayuda de otras personas, observándolas, imitándolas, siguiendo sus instrucciones o colaborando con ellas. La enseñanza eficaz es la que parte del nivel de desarrollo efectivo del estudiante, pero no para acomodarse, sino para hacerle progresar a través de la zona de desarrollo próximo, para ampliar y para generar, eventualmente, nuevas zonas de desarrollo próximo.

Finalmente plantea que la clave no se encuentra en si el aprendizaje escolar ha de conceder prioridad a los contenidos o a los procesos, contrariamente a lo que sugiere la polémica usual, sino en asegurarse que sea significativo.

\section{Principios pedagógicos}

Díaz (2006: 19), expresa que hay muchas corrientes pedagógicas que utilizan la teoría constructivista. Lo que aprenden lo hacen mediante la experimentación y no porque se les explique lo que sucede. Se deja hacer sus propias inferencias, descubrimientos y Conclusiones. También acentúa que el aprender no es un proceso de "todo o nada" sino la nueva información que se les presenta, construyendo sobre el conocimiento que ya poseen.

\section{Formación actitudes morales}

Zavala (2007:139), ha explicado que la pedagogía de los valores es un proceso sistemático profundo, donde el representante a enseñarlo ya sea padre, madre o profesor dirija las acciones del proceso hacia el fin propuesto, para ello necesita una orientación en el contenido y en la metodología para su aplicación. Plantea además, que la línea central metodológica de la teoría de la formación de valores radica en que los valores no se pueden imponer como normas externas, sino como un proceso negociador donde el niño o la niña estudiante reciba, asimile e integre a sus accionas cotidianas los valores negociados con la generación adulta.

Otro aspecto importante que señala Zavala (2007:147) es que la escuela por sí sola, no puede resolver el problema de la formación de valores, sino que es un problema social central y todos los estratos sociales tienen que ponerse en función de lo que necesita precisamente la sociedad.

Arnau (2007:49), destaca finalmente que los valores se forman en la vida familiar, en la escuela y en el entorno en el cual se desarrolla. Añade que la familia es la cuna de todos los valores positivos y negativos y se puede considerar el centro donde se refugian todas las tradiciones, es precisamente en la familia donde el niño se pone en contacto con los primeros valores y estos dejan una profunda huella. 
Retomando a Zavala (2007:59), se destaca que en el proceso docente educativo para la formación y desarrollo de valores es necesario cumplir una serie de requisitos tales como:

- Propiciar conductas positivas.

- Entender las conductas negativas.

- Escuchar antes de criticar.

- Evitar los prejuicios.

- Ser ejemplo.

- Crear un clima cooperativo de trabajo.

- Explicar los valores a alcanzar.

- Resaltar los valores patrios.

- Aprender a auto evaluarse.

- Formar convicciones.

\section{Materiales y métodos}

Este estudio es de tipo cualitativo, analítico y de corte transversal. El proceso abarcó el segundo semestre del año lectivo 2009.

El universo incluye 30 estudiantes del 5 to. grado, además a la docente, la directora del centro, el técnico del MINED y 10 padres y madres de familia que tienen sus hijos estudiando en el 5to. grado. El universo en total fue de 43 personas. Participaron como informantes claves de la investigación las estudiantes, los estudiantes, madres y padres de familia, el docente que imparte la asignatura, el técnico que atiende el centro escolar y la directora del centro.

\section{Resultados y discusión}

\section{Identificación de los diferentes contenidos del área de Persona, Cultura y Naturaleza}

Mediante la revisión del programa y texto se constató que la estructura de las áreas y sub áreas curriculares para Educación Primaria, comprende para el área curricular: Persona, Cultura y Naturaleza, y como sub-áreas: Historia y Sociedad, Medio Ambiente, Ciencia y Tecnología.

En entrevista realizada a docentes y director, estos expresan que los contenidos del texto están en idioma español, lenguaje oficial de la República de Nicaragua y 
en lengua miskitu, según expresaban los docentes, la orientación recibida en los talleres de capacitación sobre esta área es desarrollar los contenidos del texto en el idioma o lengua, en que aparece en el libro de texto de 5to grado. Así también señalan que es notoria la ausencia de temas de cultura que promueva los valores cívicos, éticos, morales y espirituales. Esto contradice el principio fundamental del área al indicar que "los contenidos de formación de actitudes morales permiten mayor comprensión entre las distintas culturas, favoreciendo la práctica de interculturalidad".

Al respecto Perrenoud (2007:53), plantea que la concreción curricular debe tener en cuenta no sólo la selección de los objetivos y contenidos, sino también la manera de planificar las actividades de aprendizaje, de forma que se ajusten a las peculiaridades de funcionamiento de la organización mental del estudiante.

\section{Análisis de las actividades pedagógicas}

Mediante entrevista realizada a los docentes de 5to. grado y el director del centro, se pudo constatar que entre las actividades pedagógicas que ejecutan los docentes para el desarrollo de la asignatura de Persona, Cultura y Naturaleza se destacan las siguientes:

- La creación de estrategias para facilitar el aprendizaje de los estudiantes.

- Motivación a los estudiantes para la realización de actividades.

- Dialogar con los estudiantes (colectiva o individualmente).

- Aclarar dudas sobre las dificultades ante determinadas actividades.

- Decorar el aula con trabajos realizados por la comunidad estudiantil.

- Realizar evaluación de los contenidos.

Los contenidos abordados en cada una de las unidades, se desarrollan mediante una serie de actividades denominadas: iniciales, actividades de desarrollo y actividades de aplicación.

- Las actividades iniciales permiten conocer el nivel de conocimiento previo que las estudiantes y los estudiantes tienen sobre el nuevo tema, y el cual servirá de referencia para iniciar adecuadamente su tratamiento, asegurando mantener activa la motivación e interés para el tratamiento de los nuevos aprendizajes (conversación-descripción de las láminas).

- Las actividades de desarrollo promueven la ejercitación, profundización y consolidación de los nuevos aprendizajes, asegura el nivel de conocimientos adquiridos sobre el tema.

- Las actividades de aplicación permiten diagnosticar la asimilación de los nuevos conocimientos, hasta dónde se comprenden o si necesitan ser reforzados; por 
consiguiente, en el proceso de facilitación de la enseñanza debe asegurarse que se realicen paso a paso todas las actividades sugeridas en el libro de texto.

\section{Valorar las buenas actitudes morales}

En la reflexión del personal docente sobre la formación de actitudes morales, se señala que a menudo se abordan temas sobre la importancia de la puntualidad, el cumplimiento de las tareas en clase y en casa, la necesidad de ayudar a sus padres en los quehaceres del hogar. También se les inculca tener cuidado cuando transitan por las calles, ya que existen personas que no tienen cuidado al conducir, situación que puede ocasionar accidentes.

El 70 por ciento de las madres y padres de familia refieren que sus hijas e hijos, apoyan con los quehaceres del hogar, son obedientes, cumplen con las obligaciones escolares haciendo sus tareas en casa. Esto concuerda con lo expresado por los estudiantes, al ilustrar que sus progenitores les aconsejan siempre, antes de retirarse de su hogar, que deben respetar a las personas mayores, a los docentes, director del centro y sobre todo respetar a sus compañeros y compañeras.

Situación que se pudo constatar mediante las observaciones realizadas en el aula de clase, en la cual la mayoría de estudiantes demostraron buena conducta. Según refiere Aranau (2007:49), la familia es la cuna de todos los valores positivos y negativos y se puede considerar el centro donde se refugian todas las tradiciones, es precisamente en la familia donde el niño se pone en contacto con los primeros valores y estos dejan una profunda huella. Concluye explicando que la escuela ofrece un tiempo, un espacio y un ámbito adecuado para el desarrollo de los valores.

Se puede concluir que los docentes, director y padres de familia están asumiendo la responsabilidad de la formación de un ciudadano de la región en condiciones de manejarse en la vida privada y pública con principios éticos y con compromiso profundo hacia el bienestar de la colectividad.

\section{Percepción sobre el área de Persona, Cultura y Naturaleza}

Los docentes de 5to. año del Centro "Marvin Michell" plantean que la metodología implementada para desarrollar el área de Persona, Cultura y Naturaleza, se basa en la participación activa y tiene como eje principal la interacción entre maestro y estudiantes, en la que una retroalimentación de conocimientos se basa en la experiencia de ambos. Parte del principio pedagógico de que el estudiante tiene un cúmulo de experiencias las que deben ser aprovechadas al máximo para innovar el proceso educativo.

Según los docentes, esta área tiene debilidad en información sobre algunos contenidos y sub-áreas. Consideran que la forma en que está estructurado actualmente el texto no es la más acertada. Ya tiene incluido la enseñanza-aprendizaje de una 
segunda lengua (L2) y la enseñanza de Lengua materna (L1). Cada una de estas formas de enseñanzas tiene sus propias metodologías.

En cuanto a la opinión de los estudiantes sobre su afinidad por esta área, el 90 por ciento coinciden en que es importante porque la profesora les enseña sobre el cuidado del medio ambiente, las plantas y el cuerpo humano. El 10 por ciento, sólo afirman que les gusta el área. Si tomamos en cuenta las respuestas dadas por los estudiantes, notamos que la mayoría hacen énfasis en la sub-área de Ciencias Naturales.

\section{Propuesta de procedimientos prácticos para la enseñanza eficaz del área Persona, Cultura y Naturaleza}

\section{Naturaleza de la asignatura:}

El área de Persona, Cultura y Naturaleza es el área que contribuye al desarrollo de la identidad personal, étnica, cultural y nacional, fortaleciendo la interacción del ser humano con la naturaleza, permitiendo la apropiación de los procesos cosmográficos, históricos, ambientales, sociales, económicos y utilización de nuevas tecnología, teniendo presente el impacto, el desarrollo sostenibles y transformación en el mundo moderno como parte del proceso de integración y globalización.

\section{Competencias del área:}

- Practicar los valores morales y espirituales en sus relaciones con los demás, respetando las diferencias culturales, equidad de género y el medio ambiente.

- Promover siempre la autoestima en diversos espacios de la vida cotidiana, la escuela, la comunidad y en la familia.

- Reconocer y valorar los procesos históricos, regionales, nacionales e internacionales.

- Práctica constante de medidas de protección y conservación del entorno natural y social de la región, país y el mundo.

\section{Ciencias de la Naturaleza y Tecnología}

1. Establece relación entre las funciones que realiza el sistema nervioso central y periférico con las actividades del cuerpo humano, enfatizando en la importancia de practicar medidas higiénicas para mantenerlo sano.

2. Reconoce la importancia de formar una familia, valorando la reproducción humana como un acontecimiento relevante en la vida.

3. Reconoce la importancia de practicar medidas higiénicas para prevenir enfermedades de transmisión sexual. 
4. Reconoce la utilidad de las propiedades de la materia en relación con las diversas actividades de la vida cotidiana.

5. Aprovecha diferentes formas y fuentes de energía en la solución de problemas de la vida cotidiana.

6. Reconoce la importancia de los componentes del universo, en relación con su vida y los avances científicos.

\section{Metodología y procedimiento para la enseñanza de la asignatura}

\section{Enseñanza del medio ambiente y los recursos naturales:}

Clase No.1

Actividades iniciales

- Para el desarrollo de este tema se recomienda realizar con la comunidad estudiantil una excursión alrededor de la escuela o de la comunidad, para que observen los distintos tipos de plantas que existen en los alrededores. Además, deberán levantar una lista de las que crecen de forma silvestre y con otras que son cultivadas.

Actividades de desarrollo

- Indagarán también la utilidad de las plantas en la comunidad y elaborarán un cuadro resumen como el siguiente:

\section{Plantas silvestres y plantas cultivadas}

Alimenticias Medicinales Ornamentales

\section{Silvestres}

\section{Cultivadas}

- Realizar comentario sobre el uso racional e irracional que se le da a la flora en especial al bosque en nuestra región.

- Elaborar con ayuda del docente una lista de medidas que podemos practicar para proteger la flora en nuestra región.

Actividades de Aplicación

- Orientar a la niñez a sembrar semillas de frijoles y de maíz para que observen diariamente el proceso de germinación y desarrollo, mientras les den el cuidado adecuado a las plantas. 


\section{CIENCIAS SOCIALES}

- Deberán de anotar en sus cuadernos de forma sistemática todas las cosas observadas durante este proceso y diariamente deberán de compartirlas en clase.

- Dibujarán una planta señalando sus partes principales, y otra destacando el proceso de fotosíntesis y la respiración de las plantas.

- Analizarán con el docente, una flor y su esquema para verificar los órganos que intervienen en la reproducción.

- Al final de cada contenido los estudiantes, compartirán sus trabajos en plenario y el docente realizará una evaluación acumulativa.

Clase No.2

Actividades iniciales:

- Utilice una dinámica para formar grupos de tres estudiantes y oriénteles que observen detenidamente la ilustración.
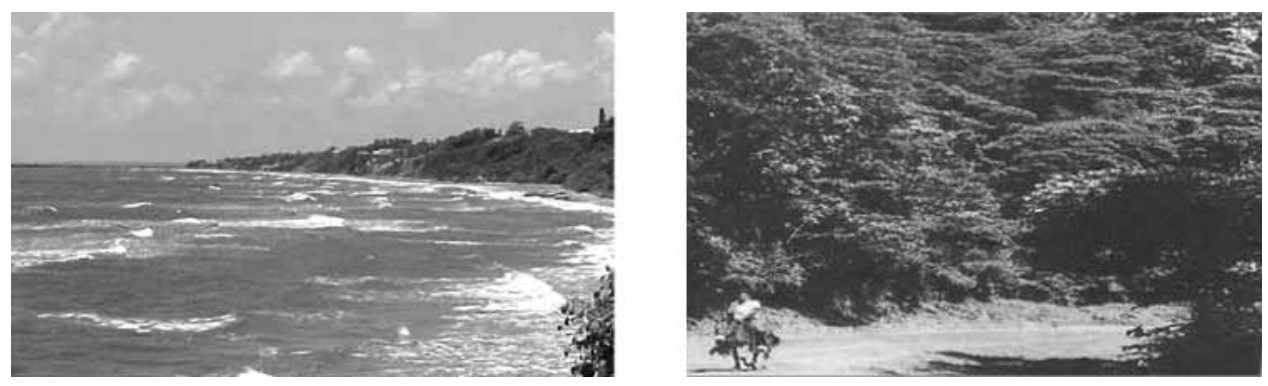

- Cuando ya han observado, pídales que conversen sobre los recursos naturales que aparecen en la lámina y la importancia que tiene para la región del Caribe en Nicaragua.

Actividades de desarrollo:

- En equipos, retome la lámina que observaron y promueva con su apoyo la elaboración de una lista acerca de los recursos naturales de la región, los clasifiquen en terrestres y acuáticos y luego los anoten en el recuadro que aparece a continuación, promueva el orden y limpieza en los trabajos.

\begin{tabular}{ll}
\hline Terrestres & Acuáticos \\
\hline 1. & 1. \\
\hline 2. & 2. \\
\hline 3. & 3. \\
\hline 4. & 4. \\
\hline 5. & 5. \\
\hline
\end{tabular}


- Aproveche los mismos equipos para orientar la elaboración en su cuaderno de una lista de principales actividades productivas de la Costa Caribe.

Actividades de aplicación:

- Oriente que cada uno de ellos y ellas conversen con las personas de su comunidad sobre el período de veda de la langosta y la tortuga verde.

- Que los estudiantes anoten en su cuaderno los comentarios y los presenten en clase.

- Con todos los contenidos trabajados en la clase y lo conversado con las personas de la comunidad, invítelos a elaborar un álbum con figuras de los principales recursos productivos y actividades propias de la región.

- Puede utilizar este álbum como la evaluación final del tema.

\section{Recursos y materiales}

Dichos materiales deben ser acordes con las posibilidades de acción. A modo de ejemplo alguno de los materiales didácticos utilizados: Conexión a Internet, libros y artículos, TV, grabador y reproductor de DVD, el pizarrón, rotafolio, fotografía, guías de aprendizaje, carteles, gráficas, ilustraciones, mapas, materiales audiovisuales.

\section{La evaluación}

La evaluación del rendimiento del estudiante se obtendrá ponderando dos factores:

En primer lugar, el grado de aprovechamiento, para lo cual se valorará la asistencia a clase - que será obligatoria y, por tanto, habrá controles-, su participación y las actividades realizadas a lo largo del año.

En segundo lugar, la nota obtenida en el examen final, que supondrá el 80 por ciento de la nota y que será necesario aprobar para superar la asignatura y tener en cuenta el otro 20 por ciento de la nota final. Este examen constará de una parte teórica y una parte práctica, que consistirá en la realización de investigaciones.

Se evaluará dentro de la evaluación continua y sumativa. Evaluaremos la adquisición de los objetivos propuestos, de los procedimientos y actitudes utilizados.

Se tendrá en cuenta las actitudes y el trabajo realizado durante las diferentes sesiones de clase, la finalidad de este tipo de unidades debe ser mejorar la convivencia en el aula y en centro, permitiendo el enriquecimiento personal de las estudiantes y los estudiantes.

Para la evaluación se utiliza la observación y el diálogo, incluyendo alguna pregunta en alguna de las pruebas de evaluación que se realicen sobre el trimestre. 


\section{Conclusiones}

Los contenidos del texto están en idioma español, lengua oficial de la República de Nicaragua y en la lengua miskitu. Según expresaban los docentes, la orientación recibida en los talleres de capacitación sobre esta área, los contenidos del texto deben ser desarrollados en el idioma o lengua, en que aparece en el libro.

Para el desarrollo de la asignatura de Persona, Cultura y Naturaleza, los docentes emplean las siguientes actividades pedagógicas: crean estrategias para facilitar el aprendizaje, motivación para la realización de actividades mediante diferentes dinámicas como canto, adivinanza, juegos, etc., dialogan de manera individual y colectivas, se aclaran dudas sobre las dificultades, se decora el aula con los trabajos realizados y se realiza la evaluación de los contenidos.

El 70 por ciento de madres y padre de familia coinciden en que apoyan con los quehaceres del hogar, sus hijos son obedientes, cumplen con las obligaciones escolares. Esto concuerda con lo expresado por los estudiantes al ilustrar que sus progenitores, les aconsejan siempre antes de retirarse de su hogar indicándoles que deben respetar a las personas mayores, a los docentes, director(a) del centro y sobre todo respetar a sus compañeras y compañeros.

En relación a los contenidos de valores permiten mayor énfasis en la comprensión entre distintas culturas, favoreciendo la práctica de la interculturalidad, mediante investigaciones con las personas mayores de la comunidad o de su hogar, etc.

Según los docentes, esta área tiene debilidad en información sobre algunos contenidos y áreas. Consideran que la forma en que está estructurada actualmente el texto no es la más acertada. Ya tiene incluido la enseñanza - aprendizaje de una segunda lengua (L2) y la enseñanza de Lengua materna (L1). No obstante, es importante señalar que cada una de estas formas de enseñanza tienen sus metodologías particulares. 


\section{Lista de referencias}

Alonso, M.J. (2007). La investigación educativa como herramienta de formación del profesorado. Edición Grao España.

Arnau, L. (2007). Once ideas claves sobre competencias de la enseñanza. Editorial Grao España.

Barboza, L. (2003). Persona, Cultura y Naturaleza. MECD. Gobierno de Nicaragua.

Díaz, F. (2006). Enseñanza situada: Vínculo entre la escuela y la vida. Edición McGrawHill. México.

Diccionario Enciclopédico Océano uno color (2004). Editorial Océano, Barcelona España.

Perrenoud, P. (2007). Diez nuevas Competencias para enseñar. $5^{\mathrm{a}}$ ed., España.

Zavala, A. et al. (2007). Cómo aprender y enseñar competencias. Editorial Grao. España. 